\title{
Change in Dislocation Structure during Cyclic Straining of Polycrystalline Aluminium at High Temperatures
}

\author{
By Tadashi Hasegawa*, Takao Yakou**, Hiroyuki Akashi*** \\ and Seiichi Karashima****
}

\begin{abstract}
Upon reversed straining of a pre-strained aluminium at high temperatures, there appears a strain region where plastic deformation proceeds at an approximately constant stress level, and the subgrain structures developed by pre-straining become loosened once and then new subgrain structures characteristic of the reversed straining are formed. In the present experiment polycrystalline aluminium specimens were subjected to a special type of cyclic straining at high temperatures where such a strain region appears.

The amount of strain and the stress level in such a strain region and the ratio of the yield stress upon stress reversal to the pre-stress level decreased with the progress of the cyclic straining. These facts suggest that the stability of dislocation structures upon stress reversal gradually increases, that is, the tendency to undergo changes due to the stress reversal is reduced as the cyclic straining progresses. Transmission electron microscopy showed that the increase in the stability of dislocation structures is caused by the decrease in density of isolated dislocations and also by the change of sub-boundaries from an unstable ill-defined state to a stable well-defined one.
\end{abstract}

(Received August 8, 1977)

\section{Introduction}

Information on the change in dislocation structures during reversed straining and/or cyclic straining is indispensable to elucidation of the dislocation behaviour in fatigue hardening or softening. Recently Mori and Fujita ${ }^{(1)}$ observed the dislocation behaviour during unloading and reversed loading of pre-strained copper at room temperature using in-situ transmission electron microscopy (TEM). They reported that isolated dislocations in the interior of cells make a backward motion

* Materials Science Division, Argonne National Laboratory, Argonne, Ill. 60439, U.S.A. On leave of absence from Department of Mechanical Engineering, Tokyo University of Agriculture and Technology, Koganei-shi, Tokyo 184, Japan.

** Department of Mechanical Engineering, Tokyo University of Agriculture and Technology, Koganei-shi, Tokyo 184, Japan.

*** Graduate School, Tokyo University of Agriculture and Technology, Koganei-shi, Tokyo 184, Japan.

**** Department of Materials Science, Tohoku University, Sendai 980, Japan.

Trans. JIM during unloading, but no remarkable change in dislocation arrangement is caused in dislocation tangles and cell walls. However, this kind of experiment has not been done at high temperatures where subgrain structures are usually formed.

On the other hand, in an effort to understand the basic principles underlying fatigue, the dislocation structure development during fatigue has been investigated by many researchers using TEM on many kinds of metals such as aluminium ${ }^{(2)}$, copper ${ }^{(3) \sim(10)}$, silver $^{(11)}$ and iron ${ }^{(12)}$. Most of these investigations have been concerned with fatigue at or below room temperature, mostly in single crystals. In reference (10), the dislocation structures in the stress-applied state were retained by pinning dislocations with a high close of fast neutrons under stress. From these studies, it is well established ${ }^{(10)(13)}$ at present that dislocation bundles formed at the initial rapid hardening stage of fatigue develop into the three-dimensional cell structures at the transition stage and at an early stage of the saturation hardening of large-strain (or largestress) amplitude fatigue, and that the cell walls have a rather regular and tidy low energy

1978 Vol. 19 
arrangement of dislocations, consisting mainly of dislocation multipoles and prismatic dislocation loops. Such cell structures may be considerably stable ${ }^{\dagger}$ for the stress reversal, because both dislocation density and cell size generally remain unchanged during the subsequent cyclic straining ${ }^{(15)}$. At the present stage of our knowledge, however, detailed processes in which dislocation structures become stable during cyclic straining are not yet clear especially in the case where fatigue softening occurs.

On the other hand, very few TEM observations have been done on dislocation structures developed during high temperature fatigue where the recovery process becomes increasingly important. Abdel-Raouf et al. ${ }^{(8)}$ and Moteff et al. ${ }^{(16)(17)}$ have recently observed the dislocation structures after fatigue at various temperatures in copper and AISI 304 and 316 stainless steels, respectively. According to their results, the size of cells (or of subgrains at high temperatures) formed during fatigue increases, and cell walls become increasingly well defined with increasing temperature. However, attention has been paid exclusively to the cell size in their works; other characteristics of dislocation structures such as the distribution of isolated dislocations and the dislocation arrangement within cell walls have not been clarified.

In previous experiments ${ }^{(18)(19)}$ deformation behaviours during reversed straining of prestrained polycrystalline aluminium were studied by tension-compression tests in a wide range of temperatures above room temperature. A strain region where the plastic deformation occurred at an approximately constant stress level was found to exist at an early stage of the reversed straining at temperatures above ' $420 \mathrm{~K}$. This region was called the "Region of

$\dagger$ Strictly speaking, some kinds of emission and/or absorption processes of dislocations may occur at cell walls during fatigue as suggested by the result of in-situ TEM $^{(1)}$. Moreover, the possibility of migration of cell walls ${ }^{(14)}$ during fatigue cannot be denied. However, essentially similar dislocation structures were observed after different loading cycles at the saturation hardening stage. Hence, in such a sense, the term "stable" or "stabilization" is used in this paper.
Constant Flow Stress (RCFS)" for convenience $^{(18)}$. It was also shown by $\operatorname{TEM}^{(19)}$ that subgrain structures developed by prestraining at high temperatures were changeable with the stress reversal: they were loosened once and then a new subgrain structure characteristic to the reversed straining was formed during the reversed straining in this region ${ }^{\dagger \dagger}$. In addition, it was found that the amount of strain in this region became smaller when the stress direction was reversed twice ${ }^{(19)}$. These facts suggest that dislocation structures tend to become more stable for the stress reversal with increasing number of the stress reversals. This work is an extension of the previous studies ${ }^{(18)(19)}$; the changes in strain and stress levels at the RCFS and also the change in dislocation structures were investigated as a function of loading cycles. The objective of this study is to obtain information on the process of stabilization of dislocation structures during cyclic straining at high temperatures.

\section{Experimental Procedure}

Aluminium polycrystalline specimens (99.99\% purity) with a gauge length of $20 \mathrm{~mm}$ and a cross section of $9 \times 10 \mathrm{~mm}^{2}$ were prepared by the same procedure as described in a previous paper ${ }^{(19)}$. The grain size was about $0.9 \mathrm{~mm}^{\dagger 3}$. The contents of main impurities by wt \% were as follows: $\mathrm{Cu}<0.001, \mathrm{Fe}<$ $0.008, \mathrm{Si}<0.002$ and $\mathrm{Mg}<0.001$.

Specimens were deformed by tensioncompression tests with an Instron-type testing machine modified for tension-compression cyclic loading. A detailed description of the machine has been given in a previous paper ${ }^{(20)}$. Mechanical testings were carried out at 458 and $503 \mathrm{~K}$ using a silicone oil bath with a strain rate of $4.2 \times 10^{-4} \mathrm{~s}^{-1}$.

†t In unidirectional deformation, such a strain region has never appeared ${ }^{(18)}$ and no dislocation rearrangement as mentioned here has been reported.

$\dagger^{3}$ Although the grain size was rather large, no problems were found in studying the Bauschinger effect on aluminium ${ }^{(20)}$. Moreover, this size was suitable for X-ray examination ${ }^{(19)}$. 


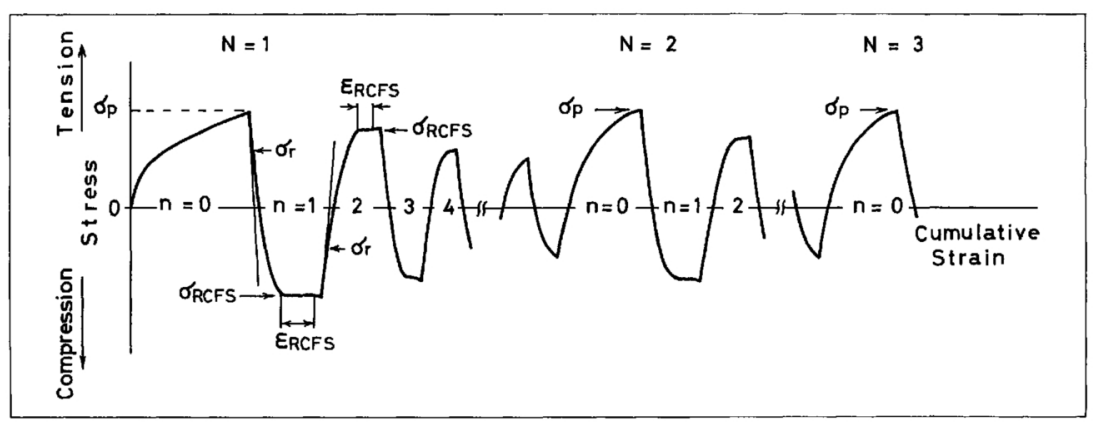

Fig. 1 Schematic stress-cumulative strain curve obtained by a special type of cyclic straining. As for the meanings of $N, n, \varepsilon_{\mathrm{RCFS}}, \sigma_{\mathrm{RCFs}}$ and $\sigma_{r}$, see text.

A schematic stress-cumulative strain curve in Fig. 1 shows how a specimen was subjected to cyclic straining in the present experiment. An annealed specimen was first deformed in tension up to a standard pre-stress level, $\sigma_{p},(N=1$ and $n=0)$, and then the loading direction was reversed from tension to compression $(N=1$ and $n=1)$. Immediately after the RCFS (an abbreviation of "Region of Constant Flow Stress") in compression was over $^{\dagger 4}$, the loading direction was again reversed from compression to tension $(N=1$ and $n=$ 2). Similar procedure was subsequently repeated. With increasing number of the stress reversals, the amount of strain in the RCFS, $\varepsilon_{\mathrm{RCFS}}$, and the stress level at this stage, $\sigma_{\mathrm{RCFS}}$, became smaller and this region finally disappeared. After the disappearance the specimen was deformed by tension once more to the standard pre-stress level, $\sigma_{p},(N=2$ and $n=0)$. Subsequent cyclic straining procedure was the same as in the case of $N=$ 1. The values of $\sigma_{p}$ were 18.0 and $12.7 \mathrm{MN} / \mathrm{m}^{2}$ at 458 and $503 \mathrm{~K}$, respectively. The yield stress in the reversed direction upon the stress reversal, $\sigma_{r}$, was determined from the stress at which the stress-strain curve deviated from the elastic straight line.

The foils for TEM were prepared by spark erosion machining and electrochemical polishing. They were examined with a Hitachi HU-11E electron microscope operating at

$\dagger^{4}$ This circumstance was well establised because the loading direction was reversed after the specimen had work-hardened by about $0.14 \mathrm{MN} / \mathrm{m}^{2}$ beyond $\sigma_{\max }$ (see the inset in Fig. 2).
$100 \mathrm{kV}$ using a $\pm 30^{\circ}$ Goniometer stage. Average thickness of the observed regions of the foils was about $0.4 \mu \mathrm{m}$. The thickness was estimated from the number of grain boundary fringes or the number of extinction contours near the edge of the foil.

\section{Experimental Results}

\section{Mechanical behaviours}

An example of stress-cumulative strain curves obtained at $503 \mathrm{~K}$ is shown in Fig. 2. As can be seen from the figure, slight softening of $0.1 \sim 0.3 \mathrm{MN} / \mathrm{m}^{2}$ was observed in the RCFS (see the enlarged stress-strain curve in the figure). Hence, the strain between two points of $\sigma_{\max }$ and the average $\sigma_{\max }$ and $\sigma_{\min }$ were respectively chosen as $\varepsilon_{\mathrm{RCFS}}$ and $\sigma_{\mathrm{RCFS}}$ (see the inset).

The changes in $\varepsilon_{\mathrm{RCFS}}$ and $\sigma_{\mathrm{RCFS}}$ with increasing number of $n$ at $503 \mathrm{~K}$ are shown in Figs. 3 and 4, respectively. In Fig. 4, the values of $\sigma_{\mathrm{RCFS}}$ obtained in both tension and compression are plotted on the same axis. From Figs. 2, 3 and 4 the following conclusions may be drawn concerning the RCFS.

(1) Values of $\varepsilon_{\mathrm{RCFS}}$ and $\sigma_{\mathrm{RCFS}}$ decrease with increasing numbers of $n$ and $N^{\dagger 5}$.

(2) Rates of decrease in both $\varepsilon_{\mathrm{RCFS}}$ and $\sigma_{\mathrm{RCFS}}$ with increasing number of $n$ are larger in the case of larger $N$.

(3) The range of $n$ over which the RCFS

$t^{5}$ The decrease in $\sigma_{\mathrm{RCFS}}$ implies that a sort of fatigue softening is occurring during the cyclic straining in each $N$ cycle. 
appears decreases with increasing number of $N$; it is expected from Fig. 3 that the RCFS will never be observed in the case of $N \geqq 10$.

(4) The stress level at which the RCFS disappears is higher in the case of larger $N$ (see the dotted line in Fig. 4).

Similar results were obtained when the specimens were subjected to cyclic straining
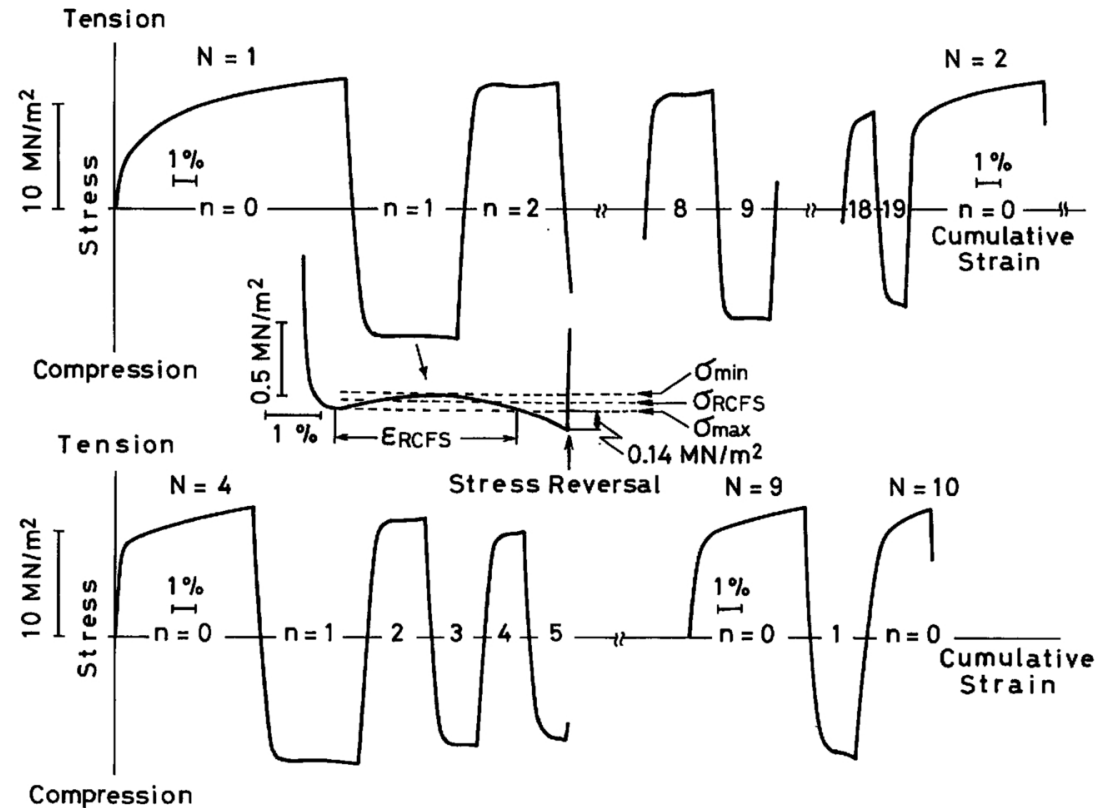

Fig. 2 Example of stress-cumulative strain curves obtained at $503 \mathrm{~K}$.

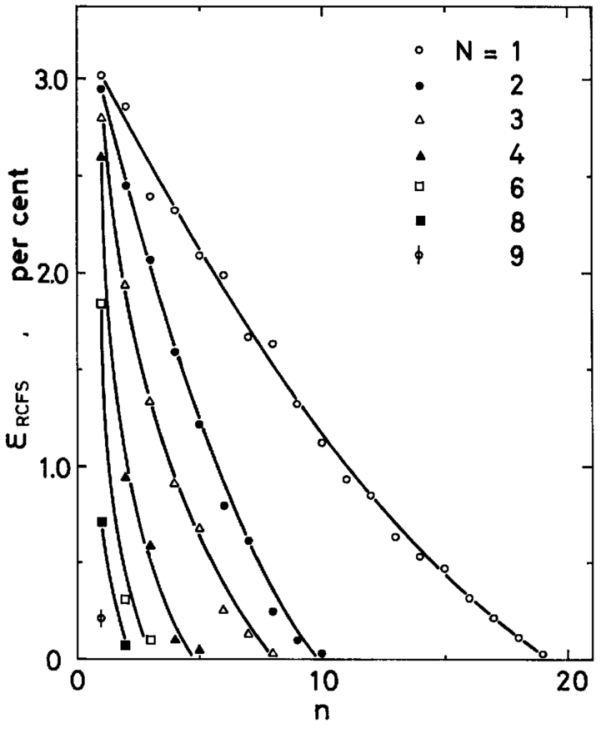

Fig. 3 Change in strain at RCFS, $\varepsilon_{\mathrm{RCFs}}$, as a function of the number of stress reversals, $n$, at $503 \mathrm{~K}$.

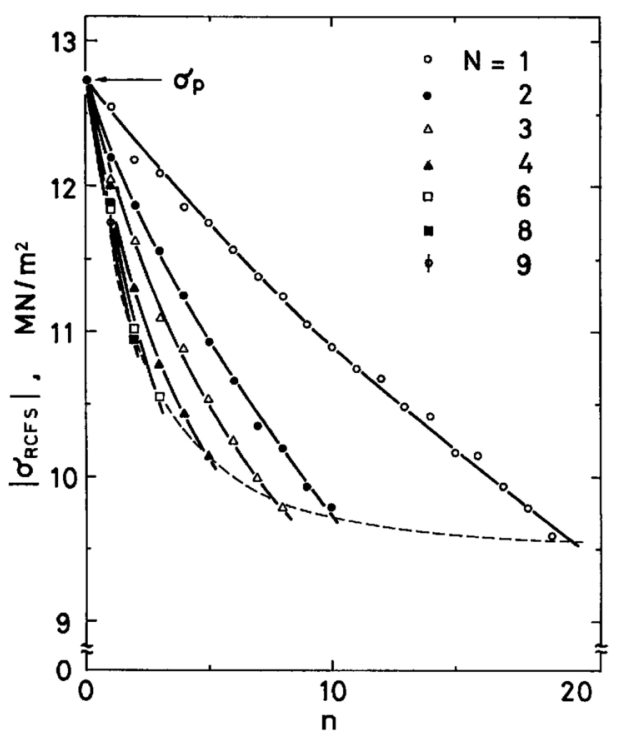

Fig. 4 Change in stress level at RCFS, $\left|\sigma_{\text {RCFs }}\right|$, as a function of the number of stress reversals, $n$, at $503 \mathrm{~K}$. 

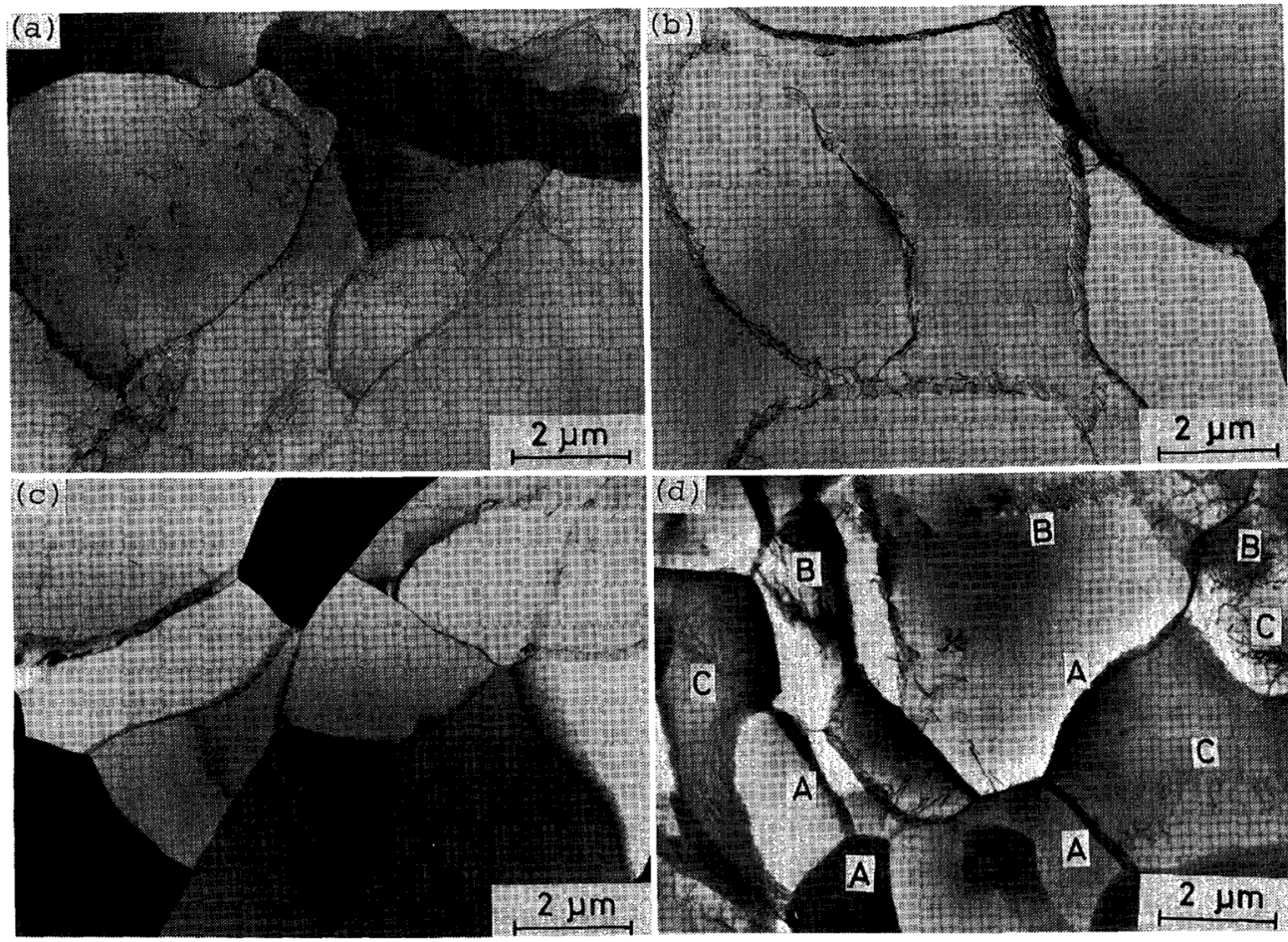

Photo. 1 Typical examples of dislocation structures at various stages of cyclic straining at $503 \mathrm{~K}$. (a) at $N=1$ and $n=0$, (b) at $N=1$ and $n=20$, (c) at $N=14$ and $n=0$, and (d) after unidirectional straining of $15 \%$ at $N=18$ and $n=0$.

at $458 \mathrm{~K}$ after a standard pre-stress of $\sigma_{p}=$ $18.0 \mathrm{MN} / \mathrm{m}^{2}{ }^{6}$ had been given. In this case, $\varepsilon_{\text {RCFS }}$ was smaller and $\sigma_{\text {RCFS }}$ was larger than those at $503 \mathrm{~K}$, respectively. Moreover, the range of $n$ over which the RCFS appears was smaller.

\section{Dislocation structures}

Thin foils were prepared from the specimens subjected to the cyclic straining up to various stages, and they were examined by TEM in order to observe the change in dislocation structures with the progress of the cyclic straining. Some differences, though not material, in the density as well as in the arrangement of dislocations were observed from grain to grain particularly at an early stage of the cyclic straining. This is perhaps because of the relatively large grain size (about $0.9 \mathrm{~mm}$ ). Hence,

$\dagger^{6}$ This level was chosen so that about the same pre-strain $(\sim 10 \%)$ might be obtained as the one in the case of $503 \mathrm{~K}$. several tens of micrographs were taken at a magnification of $5300 \times$ from more than five grains in each specimen in order to obtain comprehensive understanding of dislocation structures over the specimen.

Typical examples of dislocation structures formed at $503 \mathrm{~K}$ are shown in Photo. 1 . After the first tensile pre-straining up to $\sigma_{p}=12.7 \mathrm{MN} / \mathrm{m}^{2}$ (Photo. 1(a); $N=1$ and $n=0$ ), a subgrain structure with ill-defined sub-boundaries ${ }^{\dagger 7}$ and many isolated dislocations was observed. On the other hand, at the later stages of cyclic straining (Photo. 1(b) and (c) which were taken at stages of $N=1$

${ }^{7}$ In the present experiment it was found that the arrangement of dislocations within subboundaries became more regular at the later stage of cyclic straining. Thus, the expression "subgrain-structures with ill-defined subboundaries" is used here for the structures as shown in Photo. 1(a) instead of the "welldefined subgrain structures" in a previous paper $^{(19)}$ in order to distinguish them from the ones shown in Photo. 1(b) and (c). 
and $n=20$, and $N=14$ and $n=0$, respectively), a well-defined subgrain structure with welldefined sub-boundaries and few isolated dislocations was observed. In most of the welldefined sub-boundaries, the spacing between dislocations was so narrow that each dislocation could not be identified and the disorientation at the sub-boundaries reached several degrees at the stage of $N=14$ and $n=0$ (Photo. 1(c)).

Photograph 1(d) shows a dislocation structure in the specimen subjected to $15 \%$ tensile strain during the cyclic straining at $N=18$ and $n=0$ (namely up to a stress level of 13.4 $\mathrm{MN} / \mathrm{m}^{2}$ which is larger than the standard pre-stress level $\sigma_{p}=12.7 \mathrm{MN} / \mathrm{m}^{2}$ ). In addition to the well-defined sub-boundaries (at A), ill-defined sub-boundaries similar to those in Photo. 1(a) (at B) and many isolated dislocations (at C) can be seen.

\section{Discussion}

\section{On the softening in the region of con- stant flow stress}

In the previous experiment ${ }^{(18)}$ in which the stress direction was reversed only once on each specimen, the softening was not recognized clearly because its amount was very small. The amount showed a tendency to become somewhat large at a stage of large $n$. This is the reason why the softening was first recognized clearly in the present experiment.

Let us now consider the origin of the softening in the RCFS. The previous investigation $^{(19)}$ revealed that the decomposition and reformation of subgrain structure occurred in this region; in the middle stage of this region most of the dislocations were distributed rather uniformly forming few sub-boundaries. Furthermore, the total dislocation density, in which the dislocations within subgrains and in sub-boundaries were both included, remained almost constant throughout this region ${ }^{(19)}$. On the other hand, it was reported for a copper-aluminium solid solution ${ }^{(21)}$ that the specimen with subgrain structure showed a higher creep strength the one with uniform distribution of dislocations, if the total dislocation density was equal in both specimens.
From the facts mentioned above, it was concluded that this softening might be mainly due to the rearrangement of dislocations occurring in this region, i.e. the distribution of dislocations became uniform in the middle stage of this region.

\section{On the stabilization of dislocation struc- tures}

As mentioned in Introduction, dislocation structures formed during pre-straining are changeable against the stress reversal at an early stage of cyclic straining, and the RCFS corresponds to a strain region where dislocation structures characteristic of pre-straining change to ones characteristic of reversed straining $^{(19)}$. Hence, the results shown in Fig. 3 are considered to be due to the fact that the dislocation structures become stable for stress reversal after large numbers of $n$ and $N$.

Figure 5 shows the change in $\left(\sigma_{r}\right)_{n} / \sigma_{n}$ as a function of $n$, where $\left(\sigma_{r}\right)_{n}$ and $\sigma_{n}$ are the yield stress in the reversed direction upon the stress reversal and the pre-stress on the $n$ 'th stress reversal, respectively. As can be seen from the figure, the value of $\left(\sigma_{r}\right)_{n} / \sigma_{n}$ decreases with increasing numbers of $n$ and $N$. Since $\left(\sigma_{r}\right)_{n}$ is the stress at which plastic deformation in the reversed direction begins to occur during

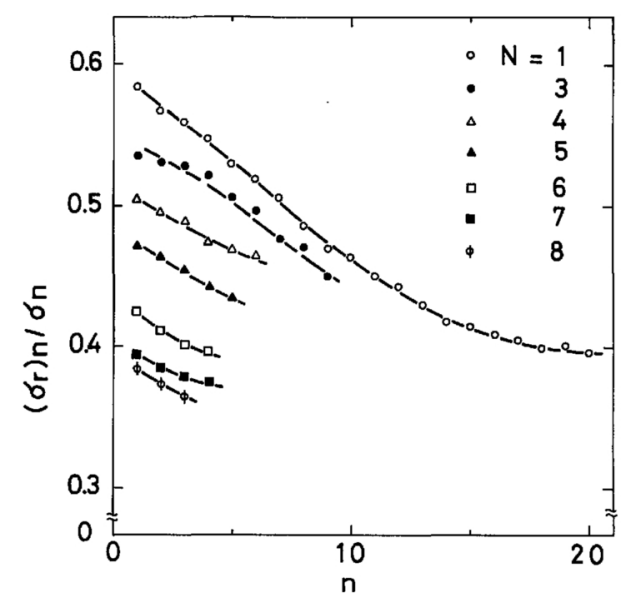

Fig. 5 Change in a parameter, $\left(\sigma_{r}\right)_{n} / \sigma_{n}$, as a function of the number of stress reversals, $n$, at $503 \mathrm{~K}$. $\left(\sigma_{r}\right)_{n}$ and $\sigma_{n}$ are yield stress in the reversed direction upon stress reversal and pre-stress on $n^{\prime}$ th stress reversal, respectively. 
unloading (see Fig. 1), the average internal stress acting against the applied stress may be estimated from it. Hence, the results shown in Fig. 5 suggest that the ratio of the average internal stress to the applied stress decreases with increasing numbers of $n$ and $N$. It is now well established that the internal stress is closely related to dislocation structures ${ }^{(21) \sim(25)}$. Thus, it may be safely concluded that the average internal stress decreases with increasing number of cycles and that dislocation structures become stable for the stress reversal after large numbers of $n$ and $N$.

\section{On the change in dislocation structures during stabilization process}

From the discussion mentioned above, the disappearance of the RCFS is considered to suggest that the dislocation structure comes to the state which is stable for both tenstion and compression. Hence, whether a dislocation structure is stable upon the stress reversal or not may be judged from the fact whether the RCFS appears or not. The following conclusions may be drawn from this point of view.

(1) Subgrain structure consisting of illdefined sub-boundaries and many isolated dislocations, as seen in Photo. 1(a), may be unstable for the stress reversal because the RCFS appears on the subsequent cyclic straining (see Figs. 2 and 3).

(2) Well-defined subgrain structure consisting of well-defined sub-boundaries and few isolated dislocations, as seen in Photo. 1(b) and (c), may be stable for the stress reversal because no RCFS appears on the subsequent cyclic straining (see Figs. 2 and 3).

(3) Reduction in the density of isolated dislocations and the change in sub-boundaries from ill-defined sub-boundaries to welldefined ones may correspond to the change in dislocation structures during the stabilization process in the cyclic straining.

After the large amount of pre-strain $(15 \%)$ at the later stage of cyclic straining $(N=18$ and $n=0$ ), ill-defined sub-boundaries (at B) and isolated dislocations (at $C$ ) were observed in addition to well-defined sub-boundaries (at A) (see Photo. 1(d)). After such a large amount of pre-strain the RCFS was clearly found to exist on the subsequent cyclic straining. Since the well-defined sub-boundaries are stable for the stress reversal, the RCFS in this case may be due mainly to the reverse motion of isolated dislocations and dislocations constituting the ill-defined subboundaries. Up to the present, however, no essential difference has been made clear between the dislocation arrangements in the illdefined and the well-defined sub-boundaries.

Let us now consider the change in subgrain size during the cyclic straining. The subgrain size in a specimen was different from grain and also from position to position even in a grain. However, it was observed that the mean subgrain size, which was estimated by the intercept method, increased by about $50 \%$ during the cyclic straing in $N=1$ (see Photo. 1(a) and (b)). This may correspond to a decrease in $\sigma_{\text {RCFs }}$ during this cyclic straing (see Fig. $4)^{+5}$. Moreover, the subgrain size at the stages of larger $N$ was considerably smaller than that at $N=1$ and $n=20$ (for example, compare Photo. 1(c) with Photo. 1(b)). This decrease in the subgrain size with the increase in $N$ may be due to the formation of new sub-boundaries during deformation up to the standard pre-stress level.

\section{Schematic representation of the stabiliza- tion process}

From the results and discussion mentioned in the foregoing sections, the changes in stress and dislocation structure during the cyclic straining may schematically be illustrated as shown in Fig. 6(a), where the vertical and horizontal directions represent the stress and dislocation structure, respectively. Tensile type and compressive type structures, which are formed by tension and compression, are unstable for compression and tension, respectively. The dislocation structure which is stable for both compression and tension is named the C-T type. Stages $A, B_{1}, C_{1}$, etc. in Fig. 6(a) correspond to the ones shown in a schematic stress-cumulative strain curve in Fig. 6(b).

When an annealed specimen is subjected to tensile pre-straining up to the standard pre- 


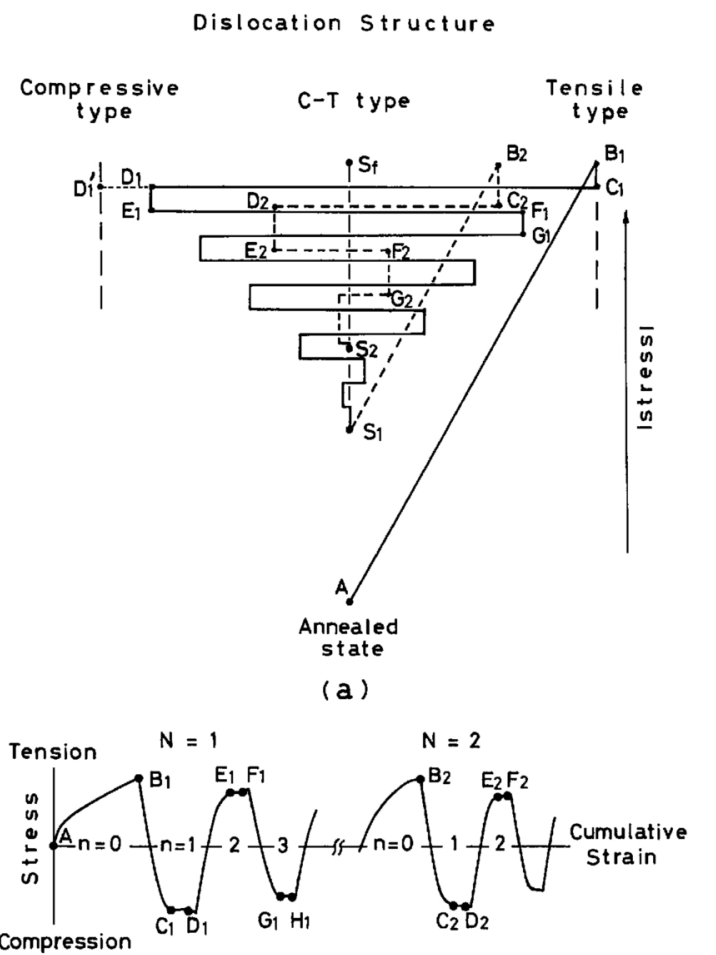

(b)

Fig. 6 Schematic representation of (a) the change in the specimen state, i.e., in dislocation structure and (b) the stress-cumulative strain curve during cyclic straining.

stress level, $\sigma_{p}\left(=12.7 \mathrm{MN} / \mathrm{m}^{2}\right.$ at $\left.503 \mathrm{~K}\right)$ $(N=1$ and $n=0)$, the dislocation structure may undergo a change from $A$ to $B_{1}$. No essential change in dislocation structure is observed during the reversed straining between points $B_{1}$ and $C_{1}{ }^{(19)}$. Stress level at the RCFS (at $C_{1}$ ) is somewhat lower than the pre-stress level at $\mathrm{B}_{1}{ }^{(18)}$. The dislocation structure changes from a tensile type to a compressive type during the RCFS, that is, from $C_{1}$ to $D_{1}$ at an approximately constant stress level. Since $\varepsilon_{\text {RCFS }}$ decreased with increasing number of $n$ (see Fig. 3), $D_{1}$ may lie closer to the C-T type than $\mathrm{C}_{1}{ }^{+8}$. With the progress of subsequent

$t^{8}$ This means tacitly that, during the deformation in the RCFS, the tensile type structure may not completely be changed into the compressive type one; that is, some effects due to pre-straining may be left behind during the reversed straining. Accumulation of such effects is considered to be closely related to fatigue hardening and/or softening phenomena. cycling, the structure may successively be changed to $E_{1}, F_{1}, G_{1}$, etc., and finally reach a stable state $S_{1}$ which is at a lower stress level than $\mathrm{B}_{1}\left(=\sigma_{p}\right)$ as shown in Fig. 6(a).

During the second tensile deformation up to $\sigma_{p}(N=2$ and $n=0)$, the tensile type structure may again be obtained as shown by point $\mathbf{B}_{2}$, which lies closer to the $C-T$ type than $B_{1}$. With the progress of the subsequent cyclic straining, the dislocation structure may undergo changes to the states $\mathrm{C}_{2}, \mathrm{D}_{2}, \mathrm{E}_{2}$, etc. and reach a stable state $\mathrm{S}_{2}$ through the same process as in $N=1$. As seen in Fig. 4, both the decreasing rate of $\sigma_{\mathrm{RCFS}}$ and the stress level at which the RCFS disappears become larger as $N$ becomes larger. Hence, stress drops in $\mathrm{B}_{2} \mathrm{C}_{2}, \mathrm{D}_{2} \mathrm{E}_{2}$, etc. are larger than those in $B_{1} C_{1}, D_{1} E_{1}$, etc. and also the stress level at $S_{2}$ is higher than $S_{1}^{\dagger 9}$. Through the same process as mentioned above, the specimen comes to the final stable state as shown by $S_{f}$ at $\sigma_{p}$; thereafter the RCFS never appears.

\section{Summary}

In the present study a relation between dislocation structure and deformation behaviour during a special type of cyclic straining was examined in polycrystalline aluminium at high temperatures where the "Region of Constant Flow Stress (RCFS)" appeared on the stress reversal. Dislocation structures were examined at various stages of cycling by transmission electron microscopy.

The amount of strain in the RCFS and the ratio of the yield stress in the reversed direction upon the stress reversal to the pre-stress level decreased with the progress of the cyclic straining. This fact suggests that dislocation structures become more stable for the stress reversal as the cyclic straining progresses.

Transmission electron microscopy has shown that ill-defined sub-boundaries developed by

$\dagger^{9}$ It has been reported for the constant-strainamplitude fatigue that cell size is inversely proportional to cyclic peak stress ${ }^{(8)(11)(26)}$. The fact that $S_{2}$ is larger than $S_{1}$ is considered to be partly due to the decrease in the subgrain size mentioned before. 
pre-straining are changeable against the stress reversal. On the other hand, well-defined subboundaries, which were observed at later stages of cycling, are considered to be stable for the stress reversal because no RCFS appeared in the subsequent cyclic straining. The density of isolated dislocations decreased with the progress of cyclic straining. These facts suggest that the reduction in the density of isolated dislocations and the change in sub-boundaries from ill-defined sub-boundaries to the well-defined ones correspond to the stabilization of dislocation structures during the cyclic straining.

\section{Acknowledgements}

The authors are grateful to Dr. Ryoji Watanabe and Mr. Toshio Kainuma, National Research Institute for Metals, Tokyo, for their help in the specimen preparation. This work was supported in part by Grant-in-Aid for Scientific Research from the Ministry of Education, Japan.

\section{REFERENCES}

(1) H. Mori and H. Fujita: J. Phy. Soc., Japan, 38 (1975), 1349.

(2) J. C. Grosskreutz: J. Appl. Phys., 34 (1963), 372.

(3) C. E. Feltner and C. Laird: Acta Met., 15 (1967), 1633.

(4) J. R. Hancock and J. C. Grosskreutz: Acta Met., 17 (1969), 77.

(5) S. J. Basinski, Z. S. Basinski and A. Howie: Phil. Mag., 19 (1969), 899.
(6) P. J. Wood: Phil. Mag., 28 (1973), 155.

(7) A. T. Winter: Phil. Mag., 28 (1973), 57.

(8) H. Abdel-Raouf, A. Plumtree and T. H. Topper: Met. Trans., 5 (1974), 267.

(9) A. Saxena and S. D. Antolovich: Met. Trans., 6A (1975), 1809.

(10) J. C. Grosskreutz and M. Mughrabi: Constitutive Equations in Plasticity, ed. by A. S. Argon, MIT Press, Cambridge, Mass. (1975), p. 251.

(11) S. M. L. Sastry, B. Ramaswami and F. Goetz: Met. Trans. 7A (1976), 243.

(12) M. Klesnil and P. Lukáš : Z. Metallk., 135 (1965), 109.

(13) J. C. Grosskreutz: Phys. Status Solidi, 47 (1971), 11.

(14) I. Imura: Electron Microscopy and Structure of Materials, ed. by G. Thomas, Univ. of California Press, Berkeley (1972), p. 104.

(15) K. Hatanaka: J. Soc. Mat. Sci. Japan, 23 (1974), 423.

(16) K. D. Challenger and J. Moteff: Met. Trans., 3 (1972), 1675.

(17) H. Nahm, J. Moteff and D. R. Diercks: Acta Met., 25 (1977), 107.

(18) T. Hasegawa and T. Yakou: Scripta Met., 8 (1974), 951.

(19) T. Hasegawa and T. Yakou and S. Karashima: Materials Sci. and Eng., 20 (1975), 267.

(20) T. Hasegawa and T. Yakou, M. Shimizu and S. Karashima: Trans. JIM, 17 (1976), 414.

(21) T. Hasegawa, Y. Ikeuchi and S. Karashima: Metal Sci. J., 6 (1972), 78.

(22) A. A. Solomon and W. D. Nix: Acta Met., 18 (1970), 863.

(23) D. J. Lloyd and J. D. Embury: Metal Sci. J., 4 (1970), 6.

(24) A. Orlová, M. Pahutová and J. Čadek: Phil. Mag., 25 (1972), 865.

(25) T. Hasegawa, S. Karashima and Y. Ikeuchi: Acta Met., 21 (1973), 887.

(26) J. E. Pratt: Acta Met., 15 (1967), 319. 